\title{
Opening the digital front door: digital offerings in a pediatric emergency department
}

\author{
Daniel Rosenfield ${ }^{1}$ (I) $\cdot$ Lianne McLean $^{1} \cdot$ Julia Clemens $^{1} \cdot$ Jason Fischer $^{1}$
}

Received: 15 July 2021 / Accepted: 24 July 2021 / Published online: 5 August 2021

() The Author(s), under exclusive licence to Canadian Association of Emergency Physicians (CAEP)/ Association Canadienne de Médecine d'Urgence (ACMU) 2021

\section{Dear CJEM,}

The journal has published numerous articles highlighting the evolving role of virtual care in emergency department care since the start of the pandemic. While early virtual care models involved appointment-based bookings for non-urgent complaints, we wanted to highlight the implementation of a scalable pediatric virtual urgent care solution. Partnering with Think Andor and utilizing Microsoft Teams and Azure, the SickKids virtual triage and urgent care is fully integrated into our enterprise-wide electronic medical record (EMR). We have created a solution that allows patients and their families to access information $24 \mathrm{~h}$ a day, 7 days a week, and access providers for a virtual visit $15 \mathrm{~h}$ per day, 7 days a week, if eligible.

After procurement and development, our digital front door was launched in April 2021. After selecting a healthcare solutions software company, we developed the symptom checker. The symptom checker is based on the SchmittThompson triage protocols, which have been externally validated [1]. The symptom checker is a simple and efficient tool used by patients and families to establish their primary question or reason for seeking care. After selecting the primary complaint, they are then offered a drop-down selection for a secondary symptom, related to and streamlined from their primary complaint. Based on their primary and secondary symptoms, they are then directed to one of three encounter outcomes-to a local emergency department for in-person care, to their primary care provider or to a virtual visit. Providers utilize a dashboard integrated into the EMR to connect with patients via secure videochat and document contemporaneously.

In the first 3 months since launch (April 7th-July 7th 2021), we have had 2875 unique encounters, resulting in $44 \%$ of patients being sent to their local ED, $28 \%$ being referred to their primary care provider and $27 \%$ being eligible for a virtual visit. The most common chief complaint was fever (24\%), followed by rash (6\%) and vomiting/diarrhea (6\%). The majority of encounters occur between the hours of 6 and 8 PM. Two thirds of users reached out to their primary care provider prior to accessing our service. A more robust qualitative and quantitative evaluation is ongoing.

Our digital front door was launched during the peak of COVID-19's third wave. A unique way to access pediatric emergency services, the system allows families to access information and disposition information at anytime, as well as care from ED providers if required.

\section{Reference}

1. Semigran H, Linder J, Gidengil C, Mehrotra A. Evaluation of symptom checkers for self diagnosis and triage: audit study. BMJ (Clin Res ed). 2015. https://doi.org/10.1136/bmj.h3480.
Daniel Rosenfield

Daniel.rosenfield@sickkids.ca

1555 University Avenue, 12th Floor, Toronto, ON M5G1X8, Canada 